\title{
Byurakan Astrophysical Observatory: 75 years of outstanding achievements
}

\author{
A. M. Mickaelian* \\ NAS RA V. Ambartsumian Byurakan Astrophysical Observatory (BAO)
}

\begin{abstract}
The Byurakan Astrophysical Observatory (BAO) is one of the most famous observatories of the Soviet Union and may be of the world. It was founded in 1946 by the outstanding scientist Viktor Ambartsumian (1908-1996) and became the leader of studies on instability phenomena in the Universe. Many discoveries have been carried out at BAO related to stars, star clusters and other systems, nebulae, galaxies and systems of galaxies. BAO has two major instruments; $1 \mathrm{~m}$ Schmidt and $2.6 \mathrm{~m}$ classical telescopes. Together with V. Ambartsumian, outstanding scientists Beniamin Markarian, Grigor Gurzadyan, Marat Arakelian and others have worked at BAO. Many important international meetings and schools for young astronomers have been held. Many important people, including State authorities, Nobel Prize Winners and others have visited BAO. BAO has statuses of RA National Value, Regional Astronomical Centre, it has UNESCO Documentary Heritage item, its garden is recognized as Dendrarium, and there is RA National Hero Viktor Ambartsumian's house-museum.
\end{abstract}

Keywords: Viktor Ambartsumian, Byurakan Astrophysical Observatory, history of science, instability phenomena

This year the National Academy of Sciences of the Republic of Armenia (NAS RA) V. Ambartsumian Byurakan Astrophysical Observatory (BAO) celebrates $75^{\text {th }}$ anniversary of its foundation. It is notable that it was founded just after the Second World War in 1946; this emphasizes the importance of the development of astronomy in Armenia by our great scientist Viktor Ambartsumian (1908-1996) who moved from the Leningrad (present Saint Petersburg) State University (LSU) to Armenia to establish the Armenian SSR Academy of Sciences together with Hovsep Orbeli (1887-1961) and other famous scientists. Orbeli was elected the President and Ambartsumian, the first Vice-President of the Academy of Sciences. However, very soon in 1947, Ambartsumian was elected the President and stayed at this position till 1993.

Though the decision at the Academy of Sciences to found an observatory and the selection of the location were made in late 1946, the construction works and first observations (with small telescopes without any building or dome) started in spring of 1947. Samvel Safaryan was appointed as the chief architect and a beautiful ensemble was designed near Byurakan village, at an average altitude of $1405 \mathrm{~m}$, including administrative buildings, laboratories, telescope towers with domes, Viktor Ambartsumian house and some auxiliary buildings. The first observations were carried out under the leadership of two experienced astronomers; Hayk Badalian (1908-1972) and Beniamin Markarian (1913-1985). The first scientific staff members were 8 researchers: Viktor Ambartsumian (Director), Hayk Badalian (Deputy Director), Beniamin Markarian, Pargev Gevorgyan, Levon Vatyan, Ruben Sahakian, Vagharshak Sanamian and Nina Ivanova. Very soon, Lyudwik Mirzoyan joined in 1947 and Grigor Gurzadian in 1948 (he was Ambartsumian's PhD student since 1944), who were not even astronomers. The Chair of Astrophysics was formally established in Yerevan State University (YSU)

*aregmick@yahoo.com 
Physics Faculty in 1944, and the first graduates (as astronomy specialists) to work at BAO appeared in 1951 (Marat Arakelian, Carlos Grigorian and Edward Khachikian). In a few years, Hrant Tovmassian, Romela Shahbazian, Arsen Kalloghlian, Elma Parsamian, Vigen Malumian and many others joined the Observatory research staff.

In 1946, the Communications of the Byurakan Astrophysical Observatory (ComBAO) were founded as the main journal for Byurakan astronomers. Ambartsumian became its first Editor-in-Chief.

It is notable that the first scientific results came immediately after the foundation of BAO. In 1947 stellar systems of new type, Stellar Associations, groups of hot (OB) giants and T Tauri stars, were discovered by V. A. Ambartsumian (Ambartsumian, 1948, 1949). It was shown for the first time that the star formation process continues at all stages of the evolution of our Galaxy, including the present one and that the star formation is a permanent process (the maximum ages of stellar associations were estimated at $10^{7} \mathrm{yr}$ compared to $10^{10} \mathrm{yr}$ of the age of the Galaxy). A conclusion was drawn that stars are formed not individually, but in groups, in stellar associations, together with gas and dust but not from them.

In November 1951, the first scientific meeting was held in BAO. Stellar Associations were the subject of the conference. The Observatory was not entirely built yet, but the investigations of Byurakan astronomers became well-known to the international astronomical community. On September 19, 1956, the official opening of BAO was celebrated by holding a conference on Non-stable stars. J. Greenstein, G. Herbig, G. Haro, E. Schatzman, P. L. Kapitsa, B. V. Kukarkin, V. V. Sobolev and other prominent scientists were among the guests.

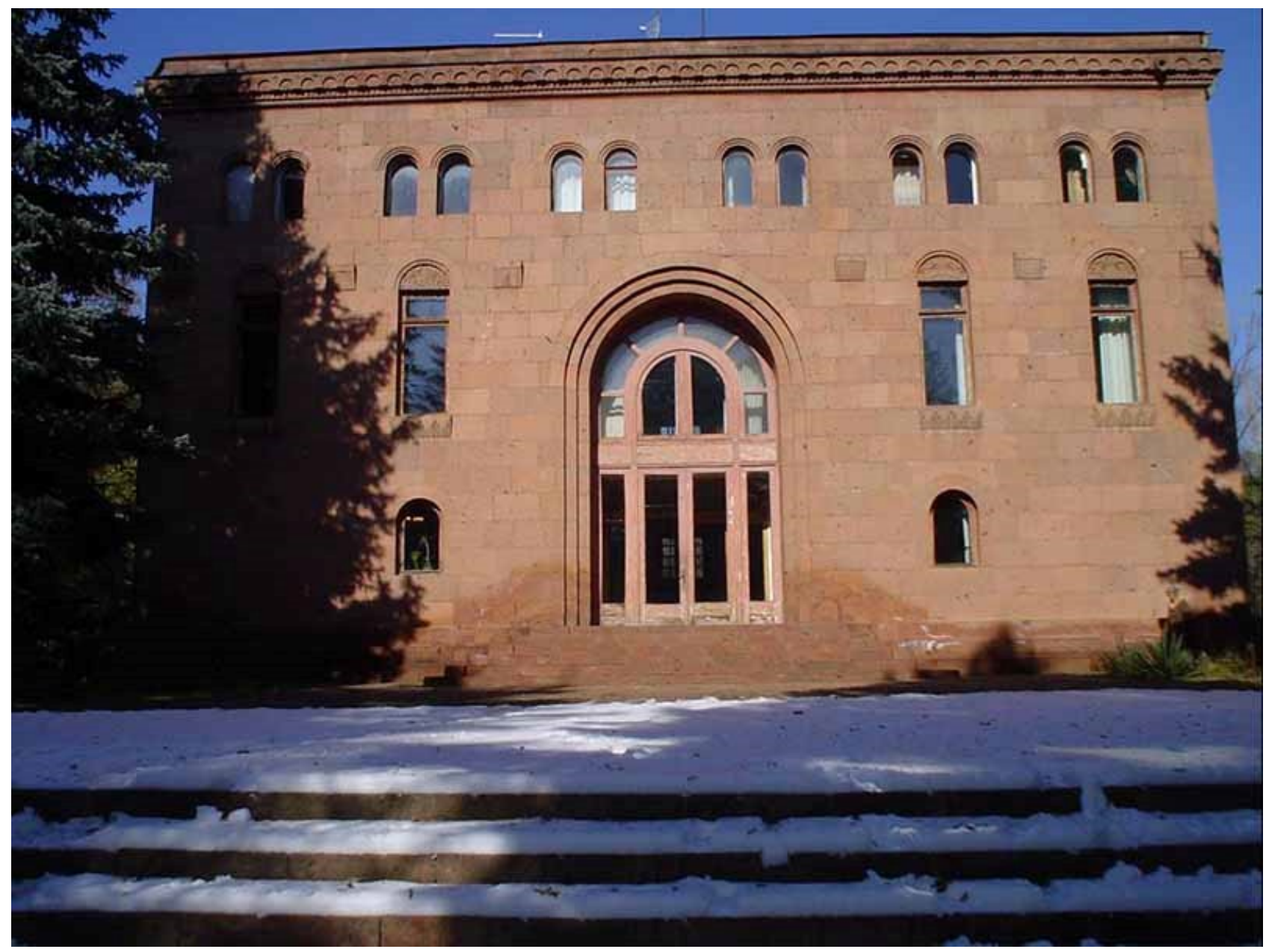

Figure 1. The Principal administrative building of BAO built in 1951.

In the mid-1950s Ambartsumian gave a new explanation for radiogalaxies radiation and proposed a new conception on the activity of galactic nuclei (Ambartsumian, 1956a,b). By the time, it was accepted by all the astronomers, and at present most of the astrophysical observatories have the sub- 
ject of Active Galactic Nuclei (AGN) as one of their main research areas. The discovery of stellar associations and Ambartsumian's idea on activity of galactic nuclei, as well as investigations on radiation transfer theory, based on Ambartsumian's Principle of Invariance (Ambartsumian, 1941, 1942), elucidated the further development of the research activities in BAO.

V. A. Ambartsumian and R. K. Shahbazian found in nearby galaxies concentrations of young stars, which they called superassociations, as well as blue companions around some galaxies, which might be thrown out from them. Later on, Shahbazian found compact groups of compact galaxies (named after Shahbazian); objects that are subject for detailed studies up to present days (Baier et al., 1974 and references therein). In early 1960s, V. A. Ambartsumian and G. S. Sahakian (from Yerevan State University, YSU) studied possible states of superdense matter and proved the possibility of existence of baryonic and hyperionic configurations with nuclear-like densities and of several Solar masses (Ambartsumian \& Saakyan, 1960, 1962a,b).

In 1960, a new Schmidt telescope with 40" (102 cm) correcting lens and 52" $(132 \mathrm{~cm})$ mirror was installed in Byurakan. Soon, in 1965, B. E. Markarian (Markarian, 1967) started a survey with a goal of revealing UV-excess galaxies (First Byurakan Survey - FBS, co-authors V. A. Lipovetski and J. A. Stepanian; Markarian et al., 1989). It was continued for 15 years and became one of the most famous surveys in modern astronomy. As a result, 1515 galaxies with UV-excess, named Markarian galaxies, were discovered. Up to now, Byurakan Schmidt is one of the largest and one of the most efficient Schmidt telescopes in the world. Markarian survey was the first systematic survey for AGN and is the largest spectroscopic survey in the world.

Observations of Markarian galaxies in Byurakan and other observatories revealed a lot of new interesting objects. E. Ye. Khachikian, together with D. Weedman (USA), discovered many new Seyferttype galaxies and for the first time made a classification of these AGN (Weedman \& Khachikyan, 1968). H. M. Tovmassian and colleagues discovered and studied the radio emission of many Markarian galaxies. More active galaxies were discovered from the lists compiled by M. A. Arakelian, who selected and published 621 galaxies with high surface brightness (Arakelian, 1975). Later on, Markarian galaxies have been studied by K. A. Sahakian, A. R. Petrosian, et al.
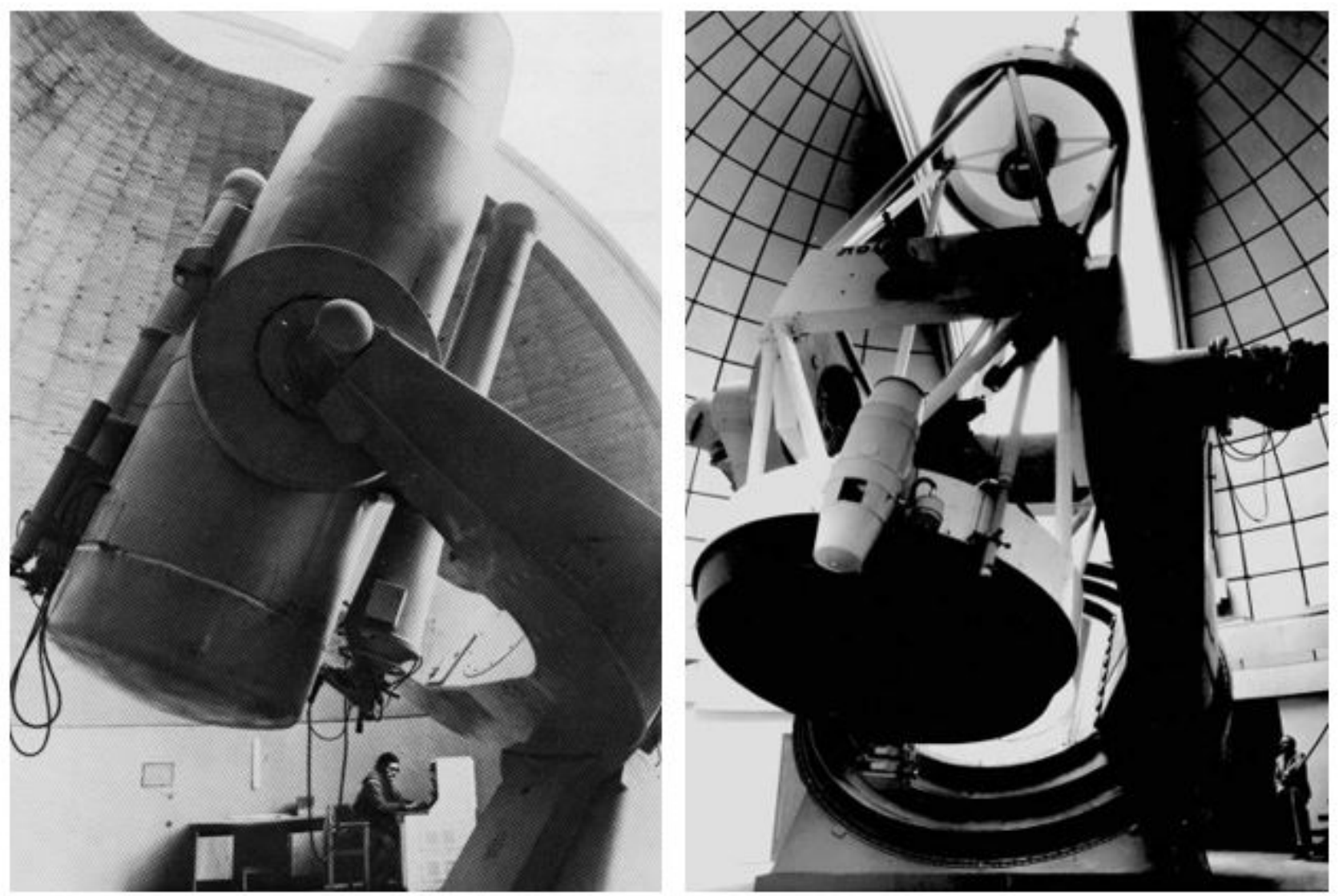

Figure 2. BAO main instruments; $1 \mathrm{~m}$ Schmidt telescope and $2.6 \mathrm{~m}$ classical reflector. 
In 1964 and 1971 conferences on Extraterrestrial Civilizations were held. The meeting of 1971 was the first international symposium on the problem of Extraterrestrial Civilizations and Communication with Them. Many prominent scientists, including astronomers, physicists, chemists, biologists, philosophers, and specialists of other related fields participated in the symposium.

In 1965 an all-Union astrophysical journal, Astrofizika (English translation: Astrophysics) was founded, and Byurakan astronomers began to publish their papers mainly in it. The Astrofizika became the main astrophysical journal of the Soviet Union as well.

IAU Symposium \#29 in May 1966 on Non-Stable Phenomena in Galaxies will remain as one of the most interesting events during the history of the observatory. J. Oort, F. Zwicky, G. Burbidge, E. Burbidge, M. Schmidt, and many other well-known scientists were present.

In 1960, H. S. Badalian carried out classification of cometary nebulae. E. S. Parsamian discovered many new cometary nebulae and published their catalogs (Parsamian, 1965, Parsamian \& Petrosian, 1979). Later on, A. L. Gyulbudaghian and T. Yu. Magakian found and studied the variability of some cometary nebulae (Giulbudagian et al., 1977). Together with colleagues, later on they discovered and studied many other young stellar objects and stellar jets.

In 1968, for its great merit to the development of science, BAO was awarded the highest prize of the Soviet Union, Lenin Order. A conference was held, devoted to V. A. Ambartsumian's 60-years anniversary. He presented a new work on statistical investigation of flare stars, estimating the real number of these objects, and predicted that all dwarf stars pass through the stage of flare activity (Ambartsumian, 1969). Beginning with the late 1960s, the investigation of flare stars became one of the main subjects of BAO. Hundreds of flare stars in star clusters and associations (Pleiades, Orion, Hyades, Praesepe, Cygnus, Coma, etc.) were discovered by L. V. Mirzoyan, E. S. Parsamian, H. S. Chavushian, L. K. Erastova, et al. Early stages of evolution of dwarf non-stable stars were investigated (Mirzoyan et al., 1981).

A project on Space Astronomy studied in Byurakan in late 1960s. Under the supervision of G. A. Gurzadyan, Byurakan scientists designed and built 2 ultraviolet "Orion" space observatories that operated onboard Soviet spacecrafts in 1971 and 1973. Later on, H. M. Tovmassian designed and built "Glazar" space observatory, which operated in 1987 onboard Soviet space station "Mir" (Tovmassian et al., 1988).

A. G. Nikoghossian, M. A. Mnatsakanian, and N. B. Yengibarian in late 1960s and 1970s obtained new solutions in Radiation Transfer Theory (RTT) with application of principle of invariance for finite thickness layers and plain-parallel medium (e.g. Nikoghossian, 1995, 2016). They also obtained new results in various fields of mathematical physics. Important contributions in theoretical astrophysics (RTT and other fields) were also made by E. Kh. Danielian, H. A. Harutyunian, G. T. Ter-Kazarian, H. V. Pikichian, R. R. Andreasyan and others.

The installation of the $2.6 \mathrm{~m}$ telescope (one of the largest telescopes in the world at that time) was rather important event in the Observatory's life. The opening of the telescope in October 1976 was accompanied by a symposium on Flare Stars. Scientific meetings on these and related objects were held in 1979 and 1984, too. The 2.6m telescope is the largest at BAO; its operations were stopped in 1991-1996 and it is again operational since 1996, when a new digital equipment was installed due to French-Armenian collaboration.

Since 1977, a specialized council for theses defenses functions. Ambartsumian was its chairman in 1977-96. The council accepts theses on astronomy, astrophysics and theoretical physics. More than 50 scientists have defended Ph.D. (Candidate) and Doctoral theses during these years.

In 1978 the Second Byurakan Survey (SBS) was conducted by B. E. Markarian, J. A. Stepanian, et al. The main goal was to obtain a homogeneous sample of quasars, emission-line and UV-excess galaxies for further cosmological investigations. More than 600 deep-limit plates were obtained during 15 years and some 3600 interesting objects were discovered (Markarian et al., 1983, Stepanian, 2005).

In October 1981, a Symposium on Principle of Invariance and its Applications was held. It was devoted to the 40th anniversary of the Principle of Invariance, Ambartsumian's theory, which found many applications in various fields of science. In 1980s two IAU symposia were organized in Byurakan. IAU Symposium \#121 on Observational Evidences of Activity in Galaxies (June 1986) gathered many outstanding astrophysicists from 17 countries. Many aspects of Ambartsumian's hypothesis and the 
classical theory of AGN were discussed. IAU Symposium \#137 on Flare Stars in Star Clusters, Associations and Solar Vicinity was held in October 1989 and gathered specialists of the corresponding field. BAO was recognized as one of the world main centres of investigations on flare stars.

In 1987 the Second Part of the First Byurakan Survey (FBS) was conducted, search and studies of the FBS stellar objects; both blue (UV excess) stellar objects (BSOs) and red (late-type) stars. It was carried out by H. V. Abrahamian, A. M. Mickaelian, and K. S. Gigoyan and resulted in discovery of 1103 BSOs (including bright QSOs, Seyferts, white dwarfs, cataclysmic variables, etc.; Mickaelian, 2008) and 1471 red stars (late M-type and C stars; Gigoyan et al., 2019). Later on, A. M. Mickaelian started a project of optical identifications based on the FBS low-dispersion spectra and revealed many galaxies and stars named Byurakan-IRAS Galaxies (BIG; Mickaelian, 1995) and Byurakan-IRAS Stars (BIS; Mickaelian \& Gigoyan, 2006).

In 1986 the Research Departments were abolished and small research groups were formed for more efficient scientific work. The Departments were re-established in 2017; at present 9 Research Departments exist like small groups, each involving a few scientists. In $1988 \mathrm{~V}$. A. Ambartsumian retired from the position of the director of the observatory, and E. Ye. Khachikian occupied it. Ambartsumian remained the honorary director of BAO until his death in 1996. In 1993-1994 H. A. Harutyunian was the acting director, in 1994-1999, the director was A. R. Petrosian, and in 1999-2003, E. Ye. Khachikian became the director for the second term, then H. A. Harutyunian was the director in 2004-2017. Since 2017, A. M. Mickaelian is the director of BAO.

After the disintegration of the Soviet Union, the Byurakan astronomers underwent difficult situation in economy and science, however, in a few years a new activity began in mid-1990s with some re-organizational process and new international collaborations. Due to French astronomers, the $2.6 \mathrm{~m}$ telescope was equipped with new instrumentation and started to give in 1996 new interesting results.

Two meetings (French-Armenian Astronomical Colloquium in 1995 and an International Symposium, devoted to the 50th anniversary of BAO in October 1996) showed that astronomers of the Observatory continue to develop Ambartsumian's ideas and have achieved new interesting results. In August 1998, the IAU Symposium \#194 on Activity in Galaxies and Related Phenomena, dedicated to Ambartsumian's 90th anniversary, was held in Byurakan. More than 100 astronomers - the most known specialists of the field from 24 countries, presented and discussed their results and prospects in this area. A new important meeting, IAU Colloquium \#184 on AGN Surveys was organized in Byurakan in June 2001. 95 scientists from 20 countries took part. The meeting was devoted to B. E. Markarian, the scientist who carried out the first systematic survey for active galaxies and opened a new era of investigations.

One of the important activities in Byurakan is the organization of Summer Schools and astronomical Olympiads (competitions for pupils and students). A number of such events were organized in 1995-2020, and the first Byurakan International Summer School (BISS) was held in August-September 2006, where 8 foreign lecturers and some 30 students participated. The next school was organized in September 2008. The 3rd one was combined with the IAU International School for Young Astronomers (ISYA) in 2010, where 18 lecturers from 7 countries and 49 students from 19 countries participated. Four more schools were organized in 2012, 2016, 2018 and 2020. BISS are being held regularly once in each two years. 7BISS was the last one.

In 2002-2007, in collaboration with Università di Roma "La Sapienza" (Italy) and Cornell University (USA) teams, the Markarian survey (First Byurakan Survey) plates were digitized and the Digitized First Byurakan Survey (DFBS; Mickaelian et al., 2007) database was created under the supervision of A. M. Mickaelian. It is one of the largest spectroscopic databases in the world and the largest astronomical database in Armenia. DFBS was the first digitization project in Armenia in all spheres. Since 2011, Markarian Survey and its Digitized version (DFBS) have been included in UNESCO "Memory of the World" International Register. Based on the DFBS and other related projects, Armenian Virtual Observatory (ArVO) was created in 2005 and was involved in International Virtual Observatories Alliance (IVOA).

In August 2007, BAO, together with the European and Armenian astronomical societies and Yerevan State Univ. (YSU), was one of the organizers of the Joint European and National Astronomy Meeting (JENAM-2007) in Yerevan. It was the largest scientific event ever organized in Armenia; 
8 parallel EAS symposia and 5 special sessions were organized. 248 scientists from 31 countries participated, and 358 plenary, invited, oral, and poster contributions were presented.

In October 2013, IAU Symposium \#304 on Multiwavelength AGN Surveys and Studies, dedicated to B. E. Markarian's 100th anniversary was held at NAS RA in Yerevan. It was the largest astronomical symposium by the number of participants (141) and represented countries (28) held in Armenia. Another large meeting, International Conference on Astronomical Heritage of the Middle East sponsored by UNESCO, was organized in Nov 2017 at NAS RA in Yerevan. This was a unique meeting representing archaeoastronomical and cultural astronomy research and results from the regional countries.

A Russian-Armenian space safety project on the monitoring of space debris was started in 2014 between BAO and Astronomical Scientific Centre related to Roskosmos, the Russian space agency. Three small telescopes were installed in Saravand (former Radioastronomy and Applied Astronomy departments location) and BAO astronomers are engaged to carry out observations led by Haik Harutyunian.

Due to obtained results the Byurakan Astrophysical Observatory is recognized by the scientific community as one of the main centres for astrophysical research. In 2013 it was recognized by the Armenian Government as National Value. The conceptions and ideas proposed in Byurakan have found their further elaboration in many observatories, a few thousands of new objects discovered in Byurakan are observed worldwide by famous astrophysicists. In 2015, BAO was recognized by IAU as one of its regional centres of astronomy for development, namely for the South-West and Central Asia.

Byurakan astronomers have participated in all large international astronomical meetings: International Astronomical Union (IAU) General Assemblies, Symposia, and Colloquia. Many of them are members of various IAU Commissions. V. A. Ambartsumian was the IAU President in 1961-64 and Vice-President in 1948-55, B. E. Markarian and E. Ye. Khachikian have been the Presidents of the IAU Commission No. 28 (Galaxies), each for 3 years. Since 2002, A. M. Mickaelian was a member of the Council of the Euro-Asian Astronomical Society (EAAS), and since 2015, he is its Vice-Chair.

In 2001, on the initiative of BAO astronomers, the Armenian Astronomical Society (ArAS) was founded, which unifies many Armenian astronomers in the world. At present 100 scientists are its members, including Armenian and other famous scientists representing 20 countries. ArAS organizes annual meetings, 12 times per year releases ArAS electronic newsletters, awards annual prize (Yervant Terzian Prize) to young astronomers, actively participates in all events organized in Byurakan, as well as maintains webpage with rich information about Armenian astronomy (including the online database of all Armenian astronomers in the world).

During the recent years, on the initiative of $\mathrm{BAO}$, collaboration has started on archaeoastronomical matters with NAS RA Institute of History, NAS RA Institute of Archaeology and Ethnography, and Matenadaran. In 2012, Armenian medieval great scientist Anania Shirakatsi's $1400^{\text {th }}$ jubilee was celebrated and an archaeoastronomical meeting was organized. BAO and ArAS jointly organized in October 2014 a meeting "Relation of Astronomy to other Sciences, Culture and Society" and together with Archaeoastronomy and Astronomy in Culture, many other interdisciplinary and multidisciplinary fields related to astronomy were discussed, including Astrochemistry, Astrobiology, Astroinformatics, etc.

BAO started another activity for collaboration between astronomers and computer scientists based on the collaboration of BAO and the NAS RA Institute of Informatics and Automation Problems (IIAP), as well as collaboration between ArVO and IVOA. BAO organized in 2015 and 2020 two international symposia on Astronomical Surveys and Big Data (ASBD and ASBD-2) related to astronomy, Big Data, computer science, Astroinformatics, etc.

The prestige of BAO was enhanced by the many-years fruitful research work and is on high level during its 75-years history. Many international scientific meetings have been held in Byurakan and dozens of astronomical institutions all over the world collaborate with BAO, including observatories and astronomical institutes of Australia, Chile, China, France, Georgia, Germany, Greece, India, Iran, Italy, Japan, Kazakhstan, Latvia, Mexico, Poland, Portugal, Russia, South Africa, Spain, UK, USA, Uzbekistan, etc. Nobel Prize winners Pablo Neruda, Subrahmanyan Chandrasekhar, Charles 
Towns, Francis Crick, Freeman Dyson, Sin-Itiro Tomonaga, Vitaly Ginzburg, John Mather and Michel Mayor, outstanding scientists J. Oort, F. Zwicky, J. Greenstein, G. Herbig, G. Haro, H. Arp, B. Bok, E. Burbidge, G. Burbidge, M. Schmidt, P. L. Kapitza, V. M. Keldish, Y. B. Zeldovich, J. Narlikar and many others and other honorary guests have visited BAO to make an acquaintance of famous astronomical centre, study its scientific program, discuss different scientific problems with Byurakan astronomers and to take part in joint investigations. State authorities, including the Soviet leaders (Nikita Khrushchov in 1961 and others), all Armenian Presidents and some Prime-Ministers, many ministers, Ambassadors of foreign countries in Armenia and others have visited BAO.

Since 1998 BAO bears the name of V. A. Ambartsumian - its founder and scientific leader. It is 75 years that $\mathrm{BAO}$ is among the world astronomical centers and successfully continues its new discoveries and high-level research.

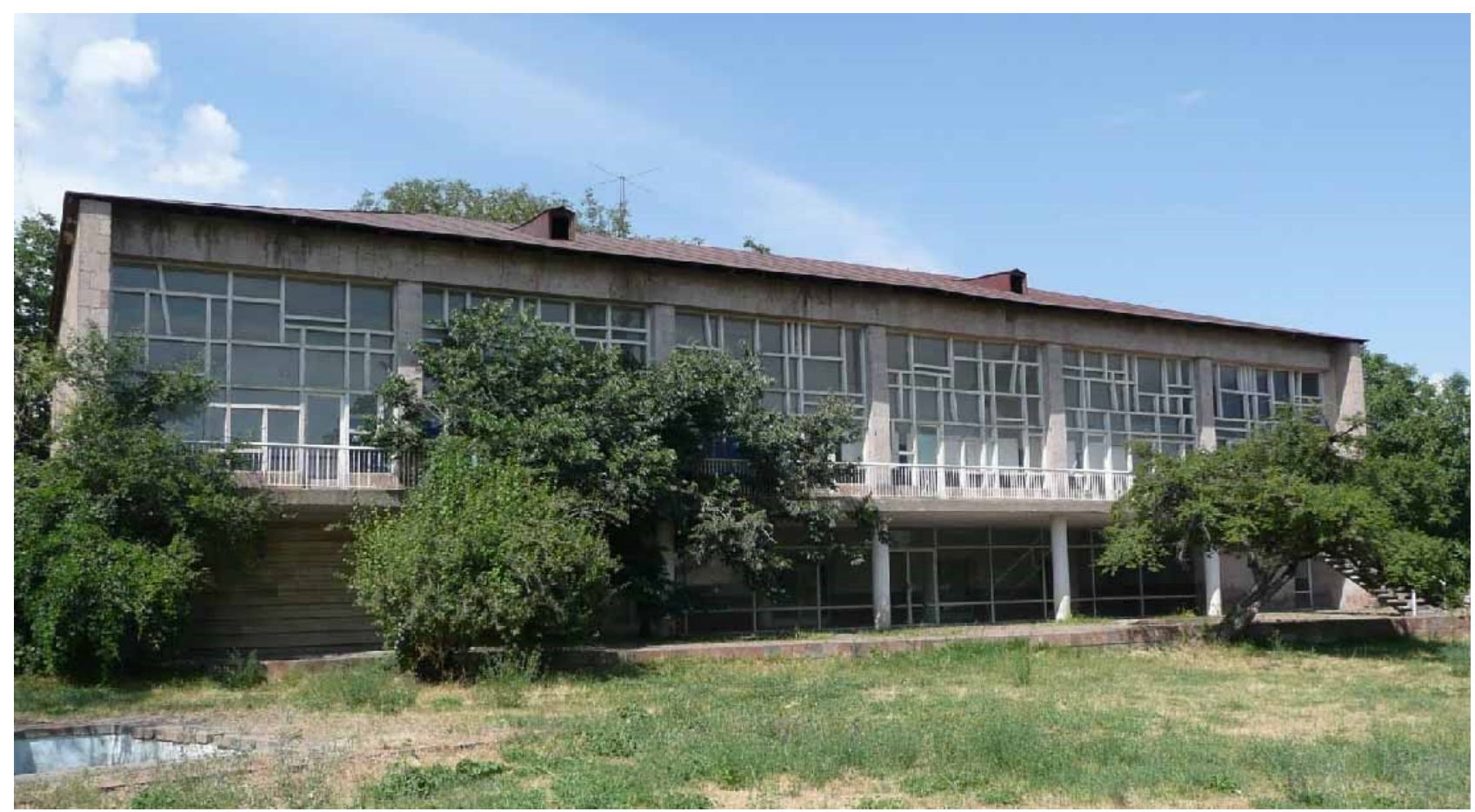

Figure 3. BAO Conference Hall, where many important international meetings were held.

Here we give the lists of most important scientific meetings held in BAO or organized by BAO (including all IAU meetings) (Table 1) and the list of the Byurakan International Summer Schools and other events for young scientists (Table 2).

Table 1: Most important scientific meetings held in BAO or organized by BAO.

\begin{tabular}{|l|l|l|}
\hline Dates & Meeting & Title / subject / description \\
\hline 1956 Sep 20-22 & BAO-1956 & $\begin{array}{l}\text { Non-Stable Stars, a Symposium Devoted to the Official } \\
\text { Inauguration of the Byurakan Astrophysical Observatory }\end{array}$ \\
\hline 1966 May 4-12 & IAU S029 & Non-Stable Phenomena in Galaxies \\
\hline 1971 Sep 6-11 & CETI-1971 & $\begin{array}{l}\text { Communication with Extraterrestrial Intelligence, } \\
\text { First International Symposium on the Problem of Extrater- } \\
\text { restrial Civilizations and Communication with them }\end{array}$ \\
\hline 1976 Oct 5-8 & ZTA-2.6 & $\begin{array}{l}\text { Flare Stars, an International Symposium Devoted to the } \\
\text { Official Opening of the 2.6m telescope }\end{array}$ \\
\hline
\end{tabular}




\begin{tabular}{|c|c|c|}
\hline 1981 Oct $26-30$ & InvPrinciple-40 & $\begin{array}{l}\text { Principle of Invariance and its Applications, an All- } \\
\text { Union Symposium Devoted to the } 40^{t h} \text { Anniversary of the } \\
\text { Principle of Invariance Introduction to the Radiation Trans- } \\
\text { fer Theory }\end{array}$ \\
\hline 1986 June $3-7$ & IAU S121 & Observational Evidence of Activity in Galaxies \\
\hline 1989 Oct $23-27$ & IAU S137 & $\begin{array}{l}\text { Flare Stars in Star Clusters, Associations and So- } \\
\text { lar Vicinity }\end{array}$ \\
\hline 1998 Aug 17-21 & IAU S194 & Activity in Galaxies and Related Phenomena \\
\hline 2001 June $18-22$ & IAU C184 & AGN surveys \\
\hline 2008 Sep 15-18 & VA-100 & $\begin{array}{l}\text { Evolution of Cosmic Objects through their Physical } \\
\text { Activity, International Conference devoted to the } 100^{t h} \\
\text { Anniversary of V. A. Ambartsumian }\end{array}$ \\
\hline 2013 Oct 7-11 & IAU S304 & Multiwavelength AGN Surveys and Studies \\
\hline 2014 Oct 7-10 & RASCS & $\begin{array}{l}\text { Relation of Astronomy to Other Sciences, Culture } \\
\text { and Society, Inter- and Multi-disciplinary Conference }\end{array}$ \\
\hline 2015 Oct $5-8$ & ASBD & $\begin{array}{l}\text { Astronomical Surveys and Big Data, International } \\
\text { Symposium dedicated to } 50^{\text {th }} \text { anniversary of Markarian } \\
\text { Survey and } 10^{\text {th }} \text { anniversary of Armenian Virtual Obser- } \\
\text { vatory (ArVO) }\end{array}$ \\
\hline 2017 Nov $13-17$ & UNESCO & $\begin{array}{l}\text { Astronomical Heritage of the Middle East, Interna- } \\
\text { tional Conference supported by UNESCO }\end{array}$ \\
\hline 2020 Sep 14-18 & ASBD-2 & $\begin{array}{l}\text { Astronomical Surveys and Big Data 2, International } \\
\text { Symposium }\end{array}$ \\
\hline 2021 Sep 20-24 & BAO-75 & $\begin{array}{l}\text { Astronomy in the Crossroads of Interdisciplinary } \\
\text { and Multidisciplinary Sciences, International Confer- } \\
\text { ence Dedicated to } 75^{\text {th }} \text { Anniversary of Byurakan Astrophys- } \\
\text { ical Observatory }\end{array}$ \\
\hline
\end{tabular}

Table 2: International schools and conferences for young astronomers held in $\mathrm{BAO}$ or organized by BAO.

\begin{tabular}{|l|l|l|}
\hline Dates & School / Conf. & Title / subject / description \\
\hline 1987 Sep 22-28 & ESO-BAO & $\begin{array}{l}\text { Observations with Large Telescopes, an International } \\
\text { School for Young Astronomers, organized jointly by ESO } \\
\text { and BAO }\end{array}$ \\
\hline 1988 Sep 27-30 & VA-80 Conf. & $\begin{array}{l}\text { Observational Evidences of Instability Phenomena } \\
\text { and their Interpretation, a Conference of Young Astro- } \\
\text { physicists Devoted to the } 80^{\text {th }} \text { Anniversary of V. A. Ambart- } \\
\text { sumian }\end{array}$ \\
\hline $\begin{array}{l}2006 \text { Aug 26- } \\
\text { Sep 3 }\end{array}$ & 1BISS & $\begin{array}{l}\text { Observational Astrophysics, } 1^{\text {st }} \text { Byurakan International } \\
\text { Summer School (1BISS) for Young Astronomers }\end{array}$ \\
\hline
\end{tabular}




\begin{tabular}{|c|c|c|}
\hline 2008 Sep 20-30 & 2BISS & $\begin{array}{l}\text { Practical Astrophysics, } 2^{\text {nd }} \text { Byurakan International Sum- } \\
\text { mer School for Young Astronomers }\end{array}$ \\
\hline $\begin{array}{l}2010 \text { Sep } 12- \\
\text { Oct } 2\end{array}$ & 3BISS/ISYA & $\begin{array}{l}3^{r d} \text { Byurakan International Summer School for Young As- } \\
\text { tronomers combined with IAU } 32^{\text {nd }} \text { International School for } \\
\text { Young Astronomers }\end{array}$ \\
\hline 2011 Nov 21-25 & Gagarin-50 & $\begin{array}{l}50 \text { Years of Cosmic Era: Real and Virtual Studies } \\
\text { of the Sky, Conference for Young Astronomers }\end{array}$ \\
\hline 2012 Sep 15-23 & 4BISS & $\begin{array}{l}4^{\text {th }} \text { Byurakan International Summer School for Young As- } \\
\text { tronomers }\end{array}$ \\
\hline 2016 Sep 12-23 & 5BISS & $\begin{array}{l}5^{\text {th }} \text { Byurakan International Summer School for Young As- } \\
\text { tronomers }\end{array}$ \\
\hline 2018 Sep 10-15 & 6BISS & $\begin{array}{l}6^{\text {th }} \text { Byurakan International Summer School for Young As- } \\
\text { tronomers }\end{array}$ \\
\hline 2019 Sep 2-6 & 1RASS & $\begin{array}{l}\text { Space Sciences and Technologies, } 1^{\text {st }} \text { Regional Astro- } \\
\text { nomical Summer School }\end{array}$ \\
\hline 2020 Sep 7-11 & 7BISS & $\begin{array}{l}\text { Astronomy and Data Science, } 7^{\text {th }} \text { Byurakan Interna- } \\
\text { tional Summer School for Young Astronomers }\end{array}$ \\
\hline 2021 Sep 13-17 & 2RASS & $2^{\text {nd }}$ Regional Astronomical Summer School \\
\hline
\end{tabular}

In Figure 4, we give the world map with collaborating with BAO countries highlighted. There are many forms of collaboration, including collaborative research projects, observing programs, organization of and participation in meetings, training of students and young researchers, etc.

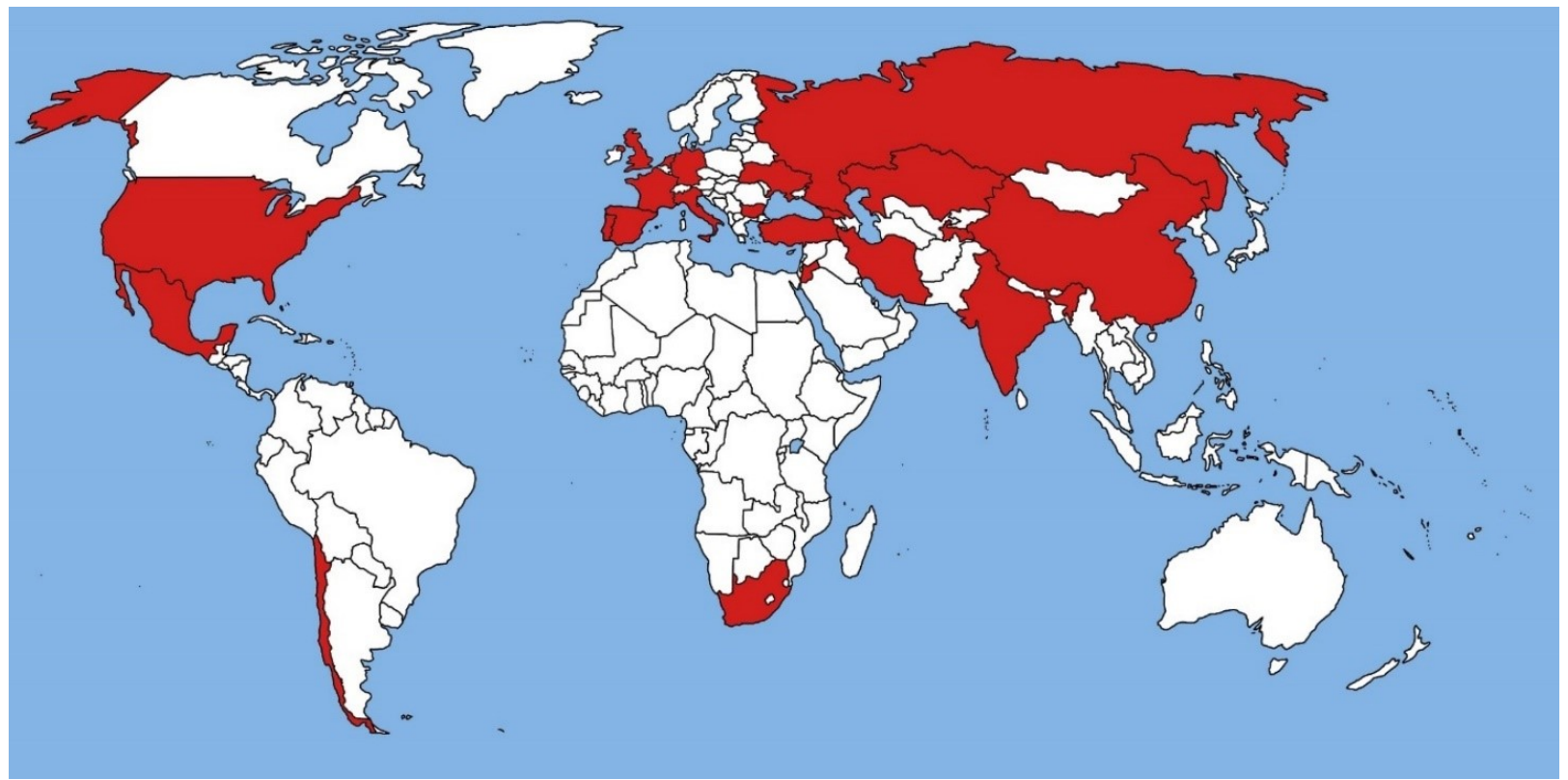

Figure 4. World map with countries collaborating with BAO.

BAO has several official statuses. We list them in Table 3. As it is obvious, most of these statuses BAO has been awarded during the last 20 years, in 2000s-2010s, which indicates about the research, educational and public activities in these years. 
Table 3. Official statuses of BAO.

\begin{tabular}{|c|c|c|}
\hline \multirow{2}{*}{ BAO official statuses } & \multicolumn{2}{|l|}{ Awarded / established } \\
\hline & Organization / body & Year \\
\hline BAO as NAS RA research institute & $\begin{array}{l}\text { National Academy of Sciences } \\
\text { of the Republic of Armenia }\end{array}$ & 1946 \\
\hline BAO as RA National Value & RA Government & 2013 \\
\hline $\begin{array}{l}\text { Armenia as IAU Regional Astronomical Centre } \\
\text { hosted by BAO }\end{array}$ & $\begin{array}{l}\text { International Astronomical } \\
\text { Union (IAU) }\end{array}$ & 2015 \\
\hline $\begin{array}{l}\text { BAO 2.6m telescope as one of the } 10 \text { biggest telescopes } \\
\text { in Europe/Asia/Africa/Australia and in the list of biggest } \\
\text { scientific instruments in USSR }\end{array}$ & USSR Academy of Sciences & 1975 \\
\hline $\begin{array}{l}\text { BAO 1m Schmidt telescope as one of the world } 10 \\
\text { biggest Schmidt telescopes }\end{array}$ & World Astronomy & 1960 \\
\hline $\begin{array}{l}\text { Markarian Survey as UNESCO "Memory of the } \\
\text { World" Documentary Heritage }\end{array}$ & UNESCO & 2011 \\
\hline $\begin{array}{l}\text { BAO among top-10 cities most often organized IAU } \\
\text { symposia and colloquia }\end{array}$ & $\begin{array}{l}\text { IAU (announced at IAU GA } \\
\text { XXX in 2018) }\end{array}$ & 2018 \\
\hline $\begin{array}{l}\text { Byurakan International Summer Schools (BISS) } \\
\text { among top-3 astronomical schools in the world }\end{array}$ & $\begin{array}{l}\text { IAU (announced at IAU GA } \\
\text { XXX in 2018) }\end{array}$ & 2018 \\
\hline $\begin{array}{l}\text { BAO was awarded Lenin Order, highest Soviet } \\
\text { award, the first among all Soviet observatories }\end{array}$ & USSR Government & 1968 \\
\hline $\begin{array}{l}\text { BAO was awarded NAS RA medal, the only among } \\
\text { all academic research institutes }\end{array}$ & NAS RA Presidium & 2021 \\
\hline $\begin{array}{l}\text { Armenian Astronomical Society (ArAS) based in } \\
\text { BAO }\end{array}$ & $\begin{array}{l}\text { European Astronomical Soci- } \\
\text { ety (EAS) affiliated society }\end{array}$ & 2001 \\
\hline $\begin{array}{l}\text { BAO project Armenian Virtual Observatory } \\
\text { (ArVO) }\end{array}$ & $\begin{array}{l}\text { International Virtual Observa- } \\
\text { tory Alliance (IVOA) member }\end{array}$ & 2005 \\
\hline $\begin{array}{l}\text { RA National Hero Viktor Ambartsumian's house- } \\
\text { museum }\end{array}$ & RA Government & 1998 \\
\hline BAO garden as Dendrarium & RA Ministry of Ecology & 2017 \\
\hline $\begin{array}{l}\text { BAO Architectural Ensemble in Armenian monu- } \\
\text { ments list }\end{array}$ & RA Ministry of Culture & 1992 \\
\hline BAO as Scientific Tourism initiator and centre & $\begin{array}{l}\text { IAU Office of Astronomy for } \\
\text { Development (OAD), Ar- } \\
\text { menian Institute of Tourism } \\
\text { (AIT) }\end{array}$ & 2016 \\
\hline $\begin{array}{l}\text { BAO Pantheon (Cemetery) as a monument of local } \\
\text { significance }\end{array}$ & $\begin{array}{l}\text { RA Ministry of Education, } \\
\text { Science, Culture and Sports }\end{array}$ & 2021 \\
\hline
\end{tabular}

Thousands of celestial objects bear Armenian names: Armenian astronomers, Byurakan, Armenia, etc. There are Markarian, Arakelian and Kazarian galaxies, Shahbazian compact groups of compact galaxies, Parsamian cometary nebulae, Gyulbudaghian-Magakian objects, First and Second Byurakan Survey objects (stars, galaxies and quasars), Byurakan-IRAS Stars (BIS) and Byurakan-IRAS Galaxies 
(BIG), Byurakan-Hamburg X-ray (ROSAT) Catalogue sources (BHRC), etc.

For more information, please see Mickaelian (2016) and visit BAO webpage at https://www. bao. am/index.php.

\section{References}

Ambartsumian V. A., 1941, Journal of Phisycs, 5, 1, 93

Ambartsumian V. A., 1942, Izvestiya Acad. Sci. USSR, Ser. Geograph. and Geophys. Sci., 3, 97

Ambartsumian V. A., 1948, Evolution of Stars and Astrophysics. Acad. Sci. ArmSSR, 39p., Yerevan

Ambartsumian V. A., 1949, Doklady USSR Acad. Sci., 68, 1, 21

Ambartsumian V. A., 1956a, Proc. Fifth conf. on Problems of Cosmogony: "Radioastronomy", held on 9-12 Mar 1955. Acad. Sci. USSR, pp 413-416

Ambartsumian V. A., 1956b, Izvestiya Acad. Sci. ArmSSR, Ser. Phys.-Math., Nat. and Tech. Sci., 9, 1, 23

Ambartsumian V. A., 1969, Proc. symp. "Stars, Nebulae, Galaxies", devoted to the 60th anniversary of academician V. A. Ambartsumian, held in Byurakan, 16-19 Sep 1968. Acad. Sci. ArmSSR, pp 283-292

Ambartsumian V. A., Saakyan G. S., 1960, Soviet Astronomy, 4, 2, 187

Ambartsumian V. A., Saakyan G. S., 1962a, Soviet Astronomy, 5, 5, 601

Ambartsumian V. A., Saakyan G. S., 1962b, Soviet Astronomy, 5, 6, 779

Arakelian M. A., 1975, Commun. BAO, 47, 3

Baier F. W., Petrosyan M. B., Tiersch H., Shakhbazian R. K., 1974, Astrofizika, 10, 327

Gigoyan K. S., Mickaelian A. M., Kostandyan G. R., 2019, MNRAS, 489, 2030

Giulbudagian A. L., Magakian T. Y., Amirkhanian A. S., 1977, Astronomy Letters, 3, 84

Markarian B. E., 1967, Astrophysics, 3, 55

Markarian B. E., Lipovetski V. A., Stepanian J. A., 1983, Astrophysics, 19, 29

Markarian B. E., Lipovetski V. A., Stepanian J. A., Erastova L. K., Shapovalova A. I., 1989, Communications of SAO, 62, 1

Mickaelian A. M., 1995, Astrophysics, 38, 4, 349

Mickaelian A. M., 2008, AJ, 136, 3, 946

Mickaelian A. M., 2016, Byurakan Astrophysical Observatory. Yerevan, Edit Print Publ. House, 64p.

Mickaelian A. M., Gigoyan K. S., 2006, Astron. Astrophys., 455, 765

Mickaelian A. M., Nesci R., Rossi C., et al. 2007, Astron. Astrophys., 464, 3, 1177

Mirzoyan L. V., Chavushyan O. S., Oganyan G. B., Ambaryan V. V., Garibdzhanyan A. T., Melikyan N. D., Natsvlishvili R. S., 1981, Astrophysics, 17, 1, 38

Nikoghossian A. G., 1995, Astrophysics, 38, 577

Nikoghossian A. G., 2016, J. Quantit. Spectrosc. Radiat. Transfer, 183, 90

Parsamian E. S., 1965, Izv. Akad. Nauk Arm. SSR., Ser. Fiz.-Math, 18, 146

Parsamian E. S., Petrosian V. M., 1979, Commun. BAO, 51, 3

Stepanian J. A., 2005, Rev. Mex. Astron. Astrofis., 41, 155

Tovmassian H. M., Khodzhayants Y. M., Krmoyan M. N., et al. 1988, Astron Letters, 14, 2, 123

Weedman D. W., Khachikyan E. E., 1968, Astrophysics, 4, 4, 243 